\title{
Finger pressure during tracking of curved contours: Implications for a visual dominance phenomenon
}

\author{
RANDOLPH D. EASTON and MICHELLE FALZETT \\ Boston College, Chestnut Hill, Massachusetts 02167
}

\begin{abstract}
An investigation was performed to determine the similarity between finger-tracking inspection of real and optically induced curved contours. It was found in the first three experiments that finger-pressure profiles produced during tracking of real curved contours were curved and opposite in orientation to the contours. This was the case whether the subjects' eyes were open or closed, provided tracking was sufficiently underway. It was also determined that the pressure profiles were the result of tracking limb-movement curves which were slightly larger in magnitude than the contours. The results of a fourth experiment indicated that pressure profiles from induced curved contours were also curved and opposite in orientation to the contours. The four experiments strengthen the contention that the experience of felt curvature under conditions of prismatic rearrangement is a joint function of vision and haptics rather than being determined exclusively by vision. A sensory conflict and a haptic system mechanism were discussed as alternatives to account for the pressure profiles obtained during finger-tracking of real- and induced-curved contours.
\end{abstract}

While the fact of visual dominance is well established (see Rock, 1975), the manner in which vision exerts its control has not been completely determined. There seems to be some agreement that vision exerts its dominant influence as a result of having privileged access to central attention (Posner, Nissen, \& Klein, 1976). What is not clear is whether conscious processing of visual information necessarily precludes processing of other sensory information. In an early account of a visual dominance phenomenon, Gibson (1933) assumed that it did. He observed that when subjects ran their forefingers back and forth along an objectively straight but visually curved contour, they reported feeling curvature in spite of the presence of veridical haptic information signaling straightness. It has since been demonstrated, however, that, in a visual-haptic conflict situation similar to Gibson's, visual curvature is not the only covariate of felt curvature (Easton, 1976). With the use of a pressure transducer, it was found that when subjects tracked an objectively straight but visually curved contour, finger-pressure profiles were curved and opposite in orientation to the visual curve. In order to produce

This research was funded in part by NIMH Grant MH2858-01 awarded to the first author. The authors wish to thank Peter Gray, Michael Numan, and Al Forsyth for helpful comments on earlier drafts of the manuscript. Michelle Falzett is now at the New School for Social Research, Program in Visual Perception. Requests for reprints should be sent to Randolph Easton, Psychology Department, Boston College, Chestnut Hill, Massachusetts 02167 . these profiles, subjects must have finger-tracked through curved paths with the same orientation as the visual curve. As an example, it was found that when subjects tracked along the inside of an optically induced convex-down contour, greatest finger pressure was applied at the middle of the contour, with the result that pressure profiles were convexup curves.

Two conclusions were drawn from the results of that investigation. First, since covariates of felt curvature other than visual information existed, the conclusion that visual information was the exclusive determinant of felt curvature seemed unwarranted. The experience of curvature may well have been mediated by haptic information. Second, the finding that pressure profiles were opposite in orientation to the induced visual curve and experienced curves suggested the possibility that an inverse relation existed in haptics between finger-pressure change and felt curvature. This issue is an empirical one, since there is no presently available a priori expectations about the relative orientations of finger-pressure change and experienced contour curvature.

A variety of relations between finger pressure and felt curvature are conceivable. It could be argued that the two would be positively related, and that when subjects track real curves finger-pressure profiles would emerge which had the same orientation as the contour. This would, for instance, be the result if subjects finger-tracked curved contours through curved paths smaller in magnitude than the contours. Put another way, if it is assumed that magnitude of 
curvature is inversely related to radius of curvature, a curved finger-tracking path with a radius larger than that of the curved contour would result in fingerpressure profiles with the same orientation as a contour curvature (see Figure 1). However, there are reasons to believe that pressure profiles produced during tracking of real curves would not have the same orientation as a curved contour. From a motorlearning perspective, it could be argued that while tracking might at first be inaccurate and result in pressure changes, subsequent tracking would become increasingly accurate, resulting in minimal pressure change along the contour. Thus, it is conceivable that constant or flat pressure profiles would occur once tracking was sufficiently underway.

Finally, it is also possible that pressure profiles from real curves would be like those produced during tracking of optically induced curvature. If pressure profiles opposite in orientation to a curved contour occurred, it would, of course, suggest a similarity between finger-tracking inspection of optically induced and real contours. Indeed, it has been argued that touch inspection of prismatically induced curvature should not be regarded as fundamentally different from ordinary bimodal perception of curvature (see Kohler, 1962). From this perspective, bimodal perception of curvature can be viewed as constituting a continuum of conflicts ranging from relatively small (e.g., spherical aberration) to relatively large (e.g., prismatic rearrangement). Findings indicating that curvature is overestimated visually are consistent with this possibility (Coren \& Festinger, 1967; Piaget, 1969). Thus, there is reason to believe that, when real curves are tracked, finger-pressure profiles would be produced which would be opposite in orientation to the contour curvature.

The experiments reported in the present paper, while bearing on these issues, were not designed specifically to test theoretical perspectives. They were designed to determine the shape of pressure profiles produced when real-curved contours are tracked with a finger, and to compare these pressure profiles to those obtained from optically induced curves. As a shape judgment task, subjects were required to finger-track steel-rod contours which were mounted on a pressure transducer. Straight and curved contours were tracked both with their eyes open and with their eyes closed. The eyes-open condition allowed a comparison of finger-pressure profiles from real curves to those obtained with induced curves. The eyes-closed condition allowed an assessment of haptic perception proper. If, as alluded to before, the experience of felt curvature in visual-haptic conflict is not solely a function of vision but involves haptics as well, it would be important to assess haptic processing of curvature.

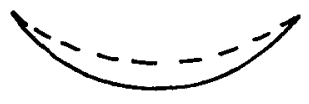

a

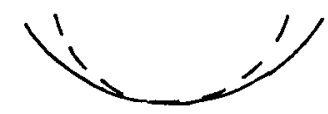

b
Figure 1. Relation between contour curvature and trackinglimb curvature. The solid lines represent contour curvatures, while the dotted lines represent tracking-limb curvatures. In Panel 1a, the radius of the tracking curve is larger than that of the contour. If subjects track curved contours in this fashion pressure would be relatively greater at the ends of the contour and would, therefore, be positively related to contour orientation. In Panel $1 \mathrm{~b}$ the radius of the tracking curve is smaller than that of the contour. Resultant finger pressure would be greatest at the middle of the contour, or inversely related to contour orientation.

Four experiments were performed. Results of the first experiment indicated that pressure profiles produced during finger-tracking of a convex-down-curved contour were opposite in orientation to the curve, whether vision was permitted or not. The second experiment was designed to determine whether the pressure profiles were actually a function of fingertracking inspection of curvature, in general, or were merely a function of the particular spatial orientation of the contour used in the first experiment. The third experiment was conducted to determine whether the pressure profiles resulted from subjects tracking through curved paths slightly larger in magnitude than the contour, or simply the application of more finger pressure at the point of greatest contour slope change. Finally, an experiment was designed to provide a direct comparison of finger pressure from induced and real curves by using real and induced contours of exactly comparable magnitude.

\section{EXPERIMENT 1}

\section{Method}

Apparatus and Materials. The tracking contours were constructed of 3/16-in. steel rods which were bent to produce curved arcs with a 37-cm chord length. A straight contour and a curved contour with a 33-mm sagitta (distance between chord midpoint and arc midpoint) were used. The radius of curvature for the curved contour was $.53 \mathrm{~m}$. The contours were glued at their ends to $3 / 8$-in. plywood blocks which were $7.5-\mathrm{cm}$ square: The curved contour was glued to the blocks in a convex-down orientation. Contours and their supporting blocks could then be interchangeably mounted into the top surface of the pressure transducer. In actuality, the top surface of the pressure transducer was a pivot board hinged along its back to a rigid vertical support and suspended by rubber strips at its front. The stiffness of the rubber suspension (see below) prevented subjects' reported awareness of movement of the board in space but at the same time allowed electromechanical monitoring of its movement. A detailed description of the pressure-transduction assembly and datarecording apparatus has been provided elsewhere (Easton, 1976).

Calibration of the pressure transducer over the range of pressures recorded during the experiment indicated that (1) a unit weight placed anywhere along straight contours resulted in equal 
displacements of the board, (2) unit increases in the weight resulted in proportional unit increases in displacement, and (3) the same equilibrium point was regained after removing any amount of displacement. As an index of pressure needed to displace the straight edge in space, a suspension-stiffness constant was computed to be equal to $4.7 \times 10^{-3} \mathrm{~mm}$ of vertical movement per gram of weight placed on the pivot board and tracking contour. ${ }^{1}$

As a control for the effect of suspension stiffness on experienced curvature, pilot investigations have been performed with different transducer suspensions. Using the method of constant stimuli, it has been found that the perceived magnitude of felt curvature of a standard contour is not effected by suspensions with stiffnesses ranging from 2 to $50 \times 10^{-3} \mathrm{~mm} / \mathrm{g}$.

A $16-\mathrm{mm}$ motion picture camera was placed $4 \mathrm{ft}$ in front of the pressure-transduction assembly such that the subject's lateral finger-tracking motion along the tracking contour and an adjacent oscilloscopic record of finger pressure were simultaneously in view. Both lateral finger-displacement position and finger-tracking pressure could be readily measured from enlarged negative images of each frame.

Subjects viewed the tracking contours monocularly (same eye as the reported dominant hand) through a glass aperture $(3 \mathrm{~cm}$ diam) mounted at eye level in one face of a 3-m cubical enclosure. The enclosure was positioned on a table and a divided curtain extending the full width of the enclosure was placed $5 \mathrm{~cm}$ beneath the viewing aperture in such a fashion that it dropped freely to the enclosure base to allow haptic exploration. The pressuretransduction assembly and tracking contours were located within the cubical enclosure at a distance of $.43 \mathrm{~m}$ from the viewing aperture. The viewing aperture restricted the field of view to the $37-\mathrm{cm}$ contour chord length. In terms of visual angle, the chord length of the contours subtended about $47^{\circ}$. The radius of the curved contour subtended $64^{\circ}$. The enclosure face opposite the viewing aperture was open so that motion picture photographs could be taken of the subject's tracking limb.

Procedure. The subject was seated in front of the cubical enclosure with his eye at the viewing aperture. He was informed that the experimenter was interested in his ability to discriminate contour shape by using a finger-tracking exploration strategy. On some inspection trials, he would be allowed to view the contour and his limb movements, while on others he would not. On each trial, he was asked to extend his dominant hand through the divided curtain of the enclosure. The experimenter placed the subject's index finger on top of the contour at its middle (inner side of the convex-down arc). The subject was instructed to begin tracking when signaled, always to his left, and to continue fingertracking back and forth along the full extent of the contour until asked to stop.

A training session was held prior to the beginning of experimental conditions. It consisted of four trials in which subjects were taught the finger-tracking exploration strategy. The subjects finger-tracked straight contours only; curved contours were not explored during practice. Half of the practice trials were performed without the aid of vision. A repetitive auditory click was presented during the practice session to cue rate of arm movement $(0.5 \mathrm{~Hz})$. The click was not presented during experimental trials as it proved distracting and tended to interrupt smooth continuous-tracking motion. The practice session lasted about $5 \mathrm{~min}$.

Experimental trials were then run. Each subject initially tiacked a straight contour, first with eyes open then with eyes closed. Curved contours were next tracked, but in an order opposite to that used for the straight contours: Curved contours were first tracked with eyes closed, then with eyes open. The first time: any subject ever tracked a curved contour, therefore, was with the eyes closed. This order of tracking trials was chosen since it was deemed necessary to determine the nature of haptic search under conditions where subjects had no previous experience with the inspected contour.
Filming began at the exact moment the subject began tracking the contour from its center and continued for two and one-quarter cycles of motion. The motion picture was taken at 14 frames/sec. For measurement purposes, the $37-\mathrm{cm}$ contour extent was divided into $103.7-\mathrm{cm}$ intervals which were clearly demarcated so that they could be included in the photographic record but were not visible to the subject. Since, on the average, the subject traversed the $37 \mathrm{~cm}$ in $1 \mathrm{sec}$ (one-half cycle), the 14 frames/sec film speed insured obtaining a pressure record for each of the 10 finger-position intervals.

The data analysis was divided into two major subdivisions, one corresponding to the first quarter cycle of tracking motion and the other corresponding to the remaining two full cycles of motion. This division was consistent with pilot study impressions which indicated that, with eyes closed, subjects' finger-pressure profiles changed in form within the first quarter cycle of motion. Once the change occurred, however, the subjects appeared to maintain the second form of tracking over many cycles of movement (up to 10 cycles were filmed during pilot investigation). As a convenience then only two full cycles were filmed during experimental trials.

Subjects. Four male and four female undergraduate students at Boston College served as volunteer subjects.

Data transformations. When an individual runs a finger back and forth along a straight contour, the pressure applied by the finger at any point occurs primarily in a direction perpendicular to the contour. The situation is different when the inside of a curved contour is tracked. At the low point of a convex-down curve, finger pressure occurs in a downward vertical direction, but as finger-tracking continues laterally in either direction with constant pressure the vertical component of the total fingerpressure vector decreases in magnitude as the horizontal component increases (see Figure 2). Since the pressure transducer used in the present experiments was sensitive to only a component of finger pressure perpendicular to the general orientation of the contour chord, a data transformation was required in order to express the data in terms of total finger pressure. Given the radius of contour curvature, the midpoint of the interval along the contour occupied by the tracking finger, and the perpendicular component of finger pressure transduced at that point, total finger pressure was computed. Expressed in this fashion, the data could be used to reflect accurately finger pressure as well as the topography of tracking-limb curvature in the frontal plane. ${ }^{2}$

Prior to analysis, the data were also expressed as deviations by subtracting pressure values across the straight contour from those (adjusted) across the curved contour. This transformation had the effect of removing from the data finger-pressure changes which occur naturally when straight contours are finger-tracked (see Easton, 1976).

\section{Results and Discussion}

Pressure deviation data from the two full cycles of tracking were first analyzed in terms of four half cycles of movement. Each half cycle consisted of the 10 intervals along the contour from initiation through termination of movement. There proved to be no effect due to half cycles of movement. In other words, the form of tracking remained essentially the same across the half cycles of movement. There were, however, differences in finger pressure along the contour, depending upon whether the tracking movement was being initiated or terminated. In addition, there were also differences attributable to whether the tracking movement occurred, roughly speaking, in a direction away from or toward the body (presumably due to the different muscle groups involved). 
While these effects are of some interest, for purposes of the present report the data were collapsed across these distinctions so that the 10 intervals along the contour corresponded to spatial positions from the left (Interval 1) to the right (Interval 10) of the subject's median plane.

Deviation data in this form are presented in the left panel of Figure 3. The double ordinate allows one to interpret the deviation profiles in terms of finger pressure or vertical displacement of the pivot board and contour. The pressure profiles from both the eyes-open and eyes-closed conditions are curved and opposite in orientation to the convex-down inspection curve. A trend analysis (Winer, 1971) indicated that both profiles contained significant quadratic components [ $\mathrm{F}$ open $(1,126)=98.34$, $\mathrm{p}<.001 ; \mathrm{F}$ closed $(1,126)=39.00, \mathrm{p}<.001]$ which did not differ significantly from one another.

Data from the first quarter cycle of tracking motion are presented in deviation form in the right panel of Figure 3. When the eyes were open, pressure began immediately to decrease, corresponding in form to pressure profiles obtained during the remaining two cycles of tracking. When the eyes were closed, pressure first increased and then decreased. A $t$ test indicated that when the eyes were closed the increase in finger pressure from Interval 1 to Interval 2 was significant $[t(7)=2.4, p<.05]$. When the eyes were open, no difference existed in finger pressure from Interval 1 to Interval 2.

Subjects' judgments of curvature can be summarized as follows. When the eyes were open, all judgments were correct. When the eyes were closed, three out of eight subjects experienced the straight contour as curved, and all eight subjects experienced the curved contour as curved.

The results indicate that when subjects inspect a convex-down curved contour with their fingers, pressure profiles occur which are opposite in orientation to the contour. This occurs whether the eyes are open or closed, provided tracking is sufficiently underway. Caution is necessary in drawing this general conclusion, however. It is important to determine whether the results represent a fundamental aspect of finger-tracking of curvature or are related spuriously to some feature of the particular stimulus curve used in the experiment. The fact that during the first quarter cycle of finger-tracking substantial pressure changes occurred is not particularly surprising, since no information regarding contour shape was available to the observer and they had previously only explored straight contours. The fact that subjects switch to a form of finger-tracking which is still inaccurate and maintain it for the duration of the inspection is more surprising. If valid, the finding represents an important characteristic of haptic exploration of curvature.

One interpretation of the obtained pressure pro-

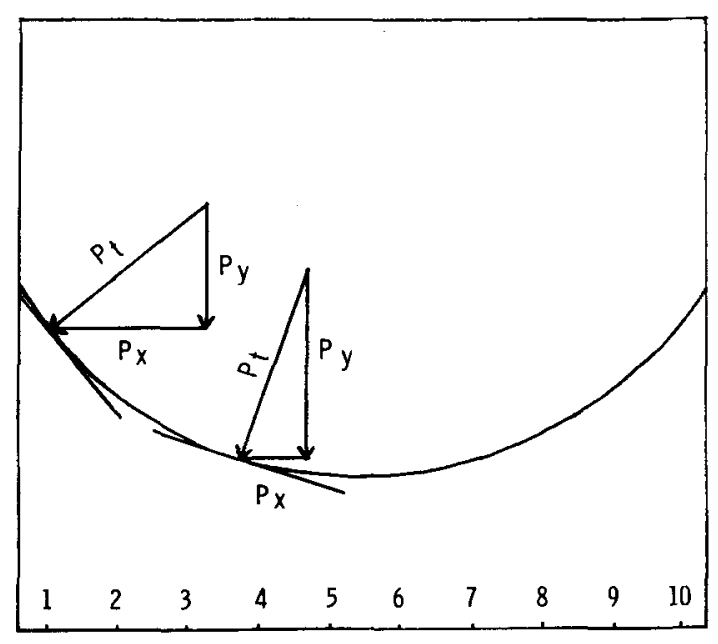

Finger Displacement Interval

Figure 2. The physics of finger-pressure magnitude when curved contours are tracked. Assuming that constant finger pressure is maintained along the contour, the transduced vertical component of pressure $\left(P_{y}\right)$ would decrease, while the horizontal component $\left(\boldsymbol{P}_{\mathbf{x}}\right)$ would increase as tracking occurred laterally in either direction from the midpoint of the curve. Given the radius of curvature, the midpoint of the interval occupied by the tracking finger, and the transduced component of pressure $\left(\mathbf{P}_{\mathbf{y}}\right)$, total finger pressure $\left(\mathbf{P}_{\mathrm{T}}\right)$ was computed.

files has been ruled out, since the data were corrected for the fact that, with the method used, the transduced vertical component of pressure would automatically decrease at the ends of a convex-down-curved contour which was tracked with constant finger pressure. It is possible, however, that with a convex-down contour the effects of gravity on the tracking finger are appreciable. It could be argued, for instance, that once tracking was underway and inertia had been overcome, the force of gravity could have resulted in greater finger pressure at the low point of the curve. The second experiment was therefore designed as a replication of the first experiment, except that the pressure transducer was turned on its end and oriented vertically rather than horizontally. Tracking contours fitted to the pivot board now had convex-left orientations, and the subjects were required to track the inside of the contour with an up-down motion of the arm. If pressure profiles from the full two cycles of motion emerged which were similar to those of Experiment 1, it would be difficult to attribute them to the effects of gravity, since the midpoint of the inspection curve would no longer be the low point.

\section{EXPERIMENT 2}

\section{Method}

Apparatus and Materials. The apparatus was identical to that used in Experiment 1 except that the pressure transducer was oriented vertically rather than horizontally. The curved contour (33-mm sagitta) when fitted to the transducer had a convex-left orientation. 

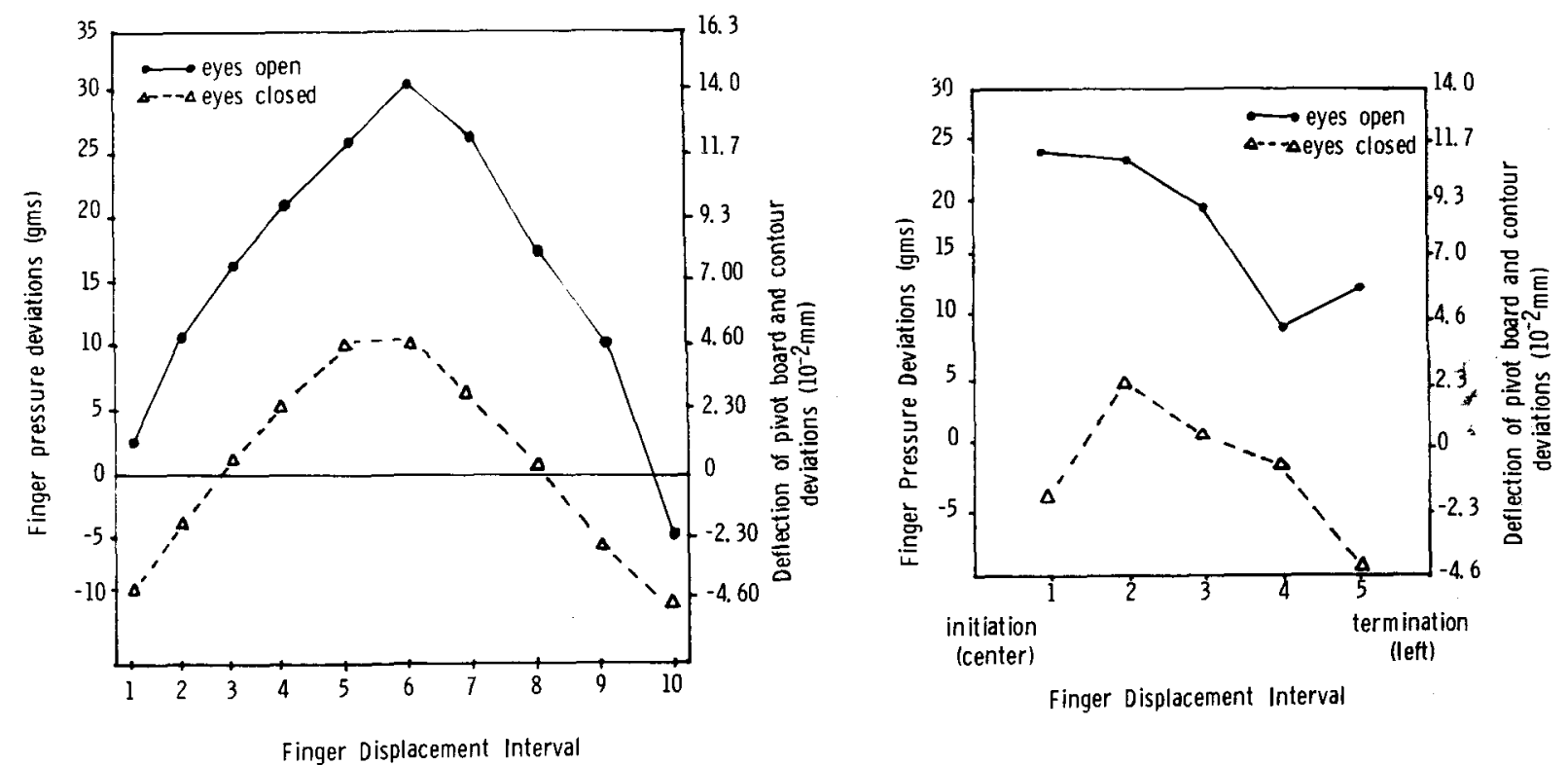

Finger Displacement Interval

Figure 3. Finger-pressure profiles and corresponding pivot-board displacement resulting from tracking the inside of a convexdown-curved contour (chord $=37 \mathrm{~cm}$; sagitta $=33 \mathrm{~mm}$ ). Data are expressed as deviations from straight-contour pressure values: The right panel represents data from the first quarter cycle of tracking and the left represents data collapsed across two additional cycles of tracking.

Procedure. The procedure was identical to Experiment 1 except that the subjects placed their forefingers on the right of the contour at its middle (inner side of arc), and initiated a tracking trial by tracking first in an upward direction and then maintaining updown tracking motion until asked to stop.

Subjects. Four male and four female undergraduate students at Boston College served as volunteer subjects.

Data transformations. The same data transformations were performed as in Experiment 1.

\section{Results and Discussion}

Pressure deviation data from the full two cyclesof-tracking motion are presented in the left panel of Figure 4 . The intervals along the abscissa correspond to spatial locations along the contour from an upward (Interval 1) to a downward (Interval 10) direction from the subjects' transverse plane. (Prior analysis of the data in another form had indicated no significant effect due to half cycles of motion, although there were effects due to whether tracking motion was being initiated vs. terminated or occurred in a direction upward vs. downward in space.) As can be seen in the figure, pressure profiles from both the eyes-open and eyes-closed conditions are curved, with greatest presssure occurring at the middle of the contour. A trend analysis indicated that both profiles contained significant quadratic components [F open $(1,126)=58.37, \mathrm{p}<.001 ; \mathrm{F}$ closed $(1,126)=58.77$, $\mathrm{p}<.001]$ which did not differ significantly from one another.

Deviation data from the first quarter cycle of tracking motion are presented in the right panel of Figure 4 . When the eyes were open, finger pressure began immediately to decrease. When the eyes were closed, finger pressure first increased and then decreased. Statistically, this finding is reflected by the fact that the eyes-closed profile contained a significant quadratic component $[\mathrm{F}(1,42)=5.6, \mathrm{p}<.025]$, while the eyes-open profile did not.

Subjects' judgments of curvature indicated the following. When the eyes were open, all judgments were correct. When the eyes were closed, one out of eight subjects experienced the straight contour as curved, and all eight subjects experienced the curved contour as curved.

The results rule out gravity as an explanation of the pressure profiles obtained in the first experiment. Finger-pressure profiles inversely related to contour curvature were obtained whether the midpoint of the contour was a low point in space or not. There are, however, two different mechanisms which could underlie the effect. The pressure profiles could have emerged as the result of tracking curvature slightly larger in magnitude than the curved contour. This would be analogous to the fact that in the inducedcurvature study limb-movement curvature was greater than contour curvature. Alternatively, it could be argued that the pressure profiles from real curves result from the application of more finger pressure at the point of greatest contour slope change. A third experiment was designed to test these two hypotheses. Subjects were required to finger-track a convex-up contour along its outer side. If subjects do, in fact, track through curves greater in magnitude than the curved contour, greatest pressure should occur at the ends of the contour. If subjects press hardest at the point of maximum stimulus change, greatest pressure should occur at the middle of the contour. 

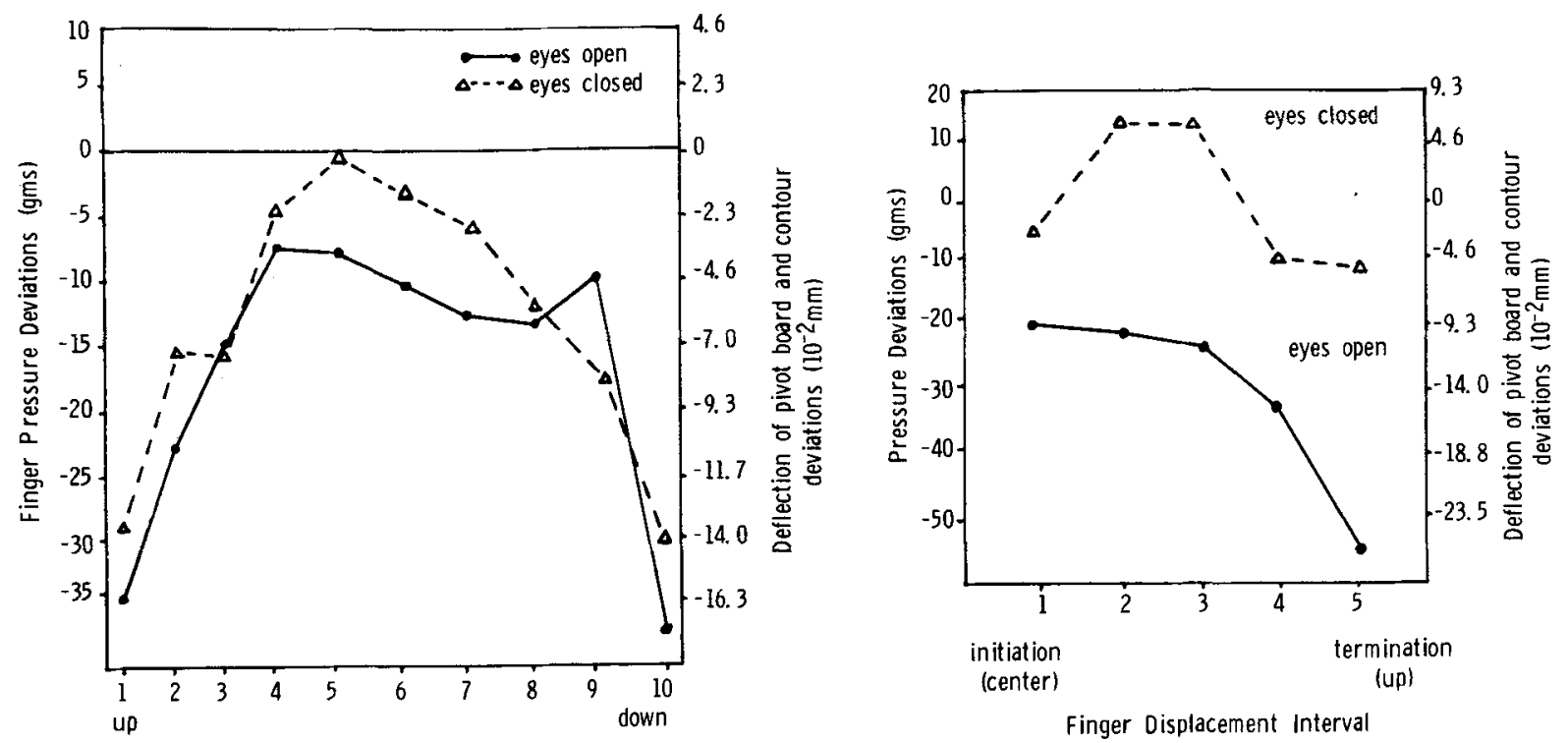

Finger Displacement Interval

Finger Displacement Interval

Figure 4. Finger-pressure profiles and corresponding pivot-board displacements resulting from tracking the inside of a convex-left curved contour (chord $=37 \mathrm{~cm}$; sagitta $=33 \mathrm{~mm}$ ). Data are expressed as deviations from straight-contour pressure values: The right panel represents data from the first quarter cycle of tracking and the left represents data collapsed across two additional cycles of tracking.

\section{EXPERIMENT 3}

\section{Method}

Apparatus and Materials. The apparatus and materials were essentially the same as those used in the first two experiments. The pressure transducer was oriented horizontally and a convex-up curved contour was used which had the same magnitude as the curves used in the previous experiments (33-mm sagitta).

Procedure. The procedure was identical to that used in Experiment 1 , except that subjects were now required to track along the outside of a convex-up arc.

Data transformations. The same transformations which were performed in Experiments 1 and 2 were again performed.

\section{Results and Discussion}

Pressure deviation from the full two cycles of movement are presented in the left panel of Figure 5. The intervals along the abscissa correspond to spatial positions along the contour from the left (Interval 1) to the right (Interval 10) of the subjects' median plane. (Preliminary analysis again indicated no effects due to half cycles of movement. There were, however, significant effects due to whether tracking movement was being initiated vs. terminated, or occurred in a direction away from vs. toward the body.) As can be seen in the figure, the pressure deviation profiles are curved, with greatest pressure occurring at the ends of the contour. A trend analysis indicated that both the eyes-open and eyes-closed profiles contained significant quadratic components [F open $(1,126)=$ $46.62, \mathrm{p}<.001$; F closed $(1,126)=91.83, \mathrm{p}<.001]$. In addition, the quadratic trend score from the eyesclosed profile was significantly larger than that from the eyes-open profile $[F(1,7)=8.47, p<.01]$.
Deviation data from the first quarter cycle of movement are presented in the right panel of Figure 5. There appears to be a tendency when the eyes were closed for pressure to decrease before increasing. $t$ tests indicated that while finger pressure had increased significantly by the fourth interval when the eyes were open $[t(7)=1.9, p<.05]$, pressure did not increase significantly until the fifth interval when the eyes were closed $[t(7)=2.5, p<.05]$.

Judgments of curvature indicated the following. When the eyes were open, all judgments were correct. When the eyes were closed, three out of eight subjects experienced the straight contour as curved, and all the subjects experienced the curved contour as curved.

In summary, the results indicate that once haptic exploration is underway, subjects finger-track through curved paths slightly greater in magnitude than the curved contour. The consequence, of course, is that pressure profiles are inversely related to contour curvature. The alternate interpretation, that subjects merely press hardest at the point of greatest stimulus change, can be ruled out, since subjects in fact produced the least amount of pressure at the middle of the outside of a convex-up curve.

The first three experiments demonstrate that fingerpressure profiles produced when real contours are tracked are opposite in orientation to the contours. The profiles result from tracking-limb curves which have the same orientation as the contour, but which are slightly larger in magnitude. The results are clearly similar to those which occur during finger-tracking of optically induced contours. However, the curved 

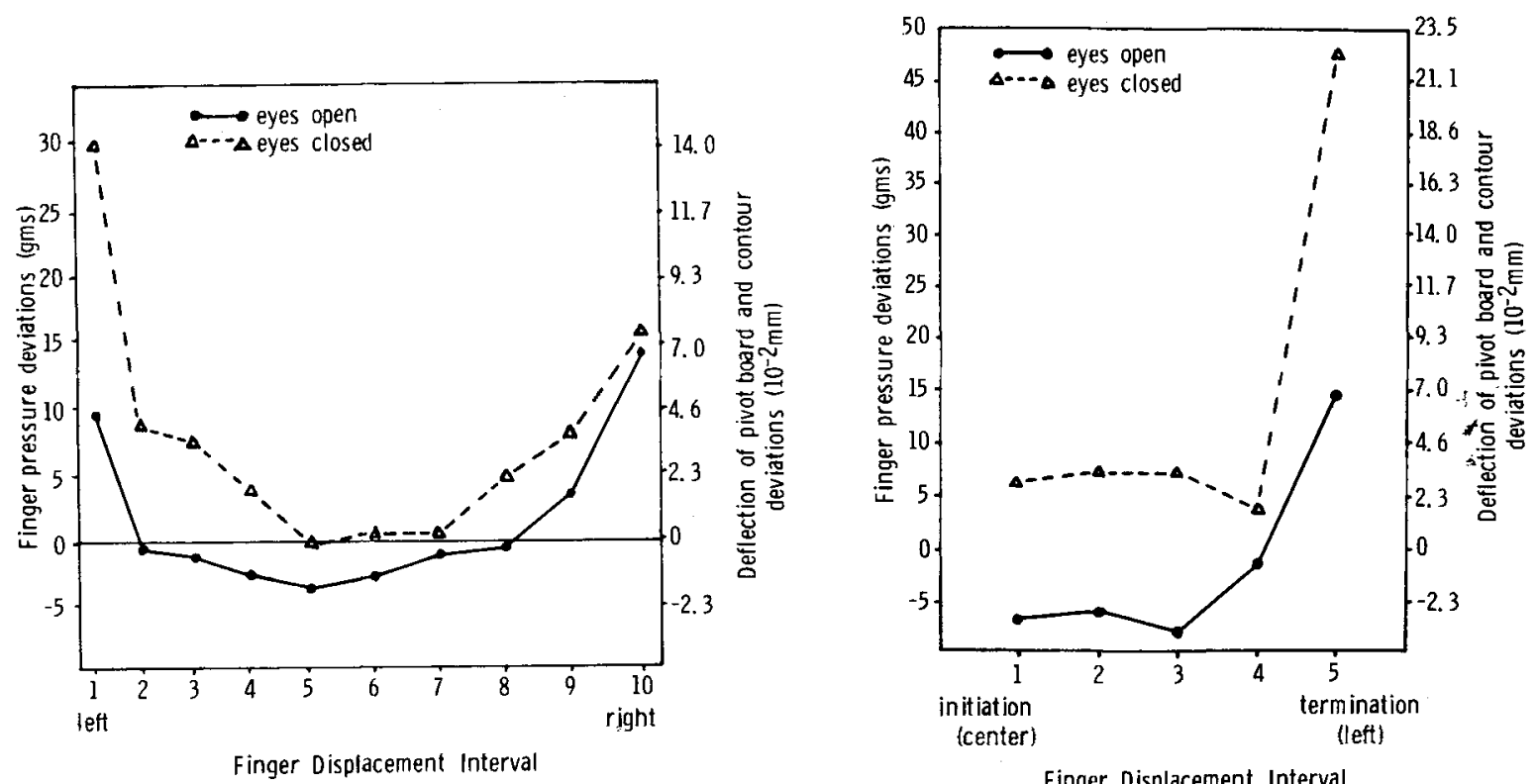

Finger Displacement Interval

Figure 5. Finger-pressure profiles and corresponding pivot-board displacement resulting from tracking the outside of a convex-up curved contour (chord $=37 \mathrm{~cm}$; sagitta $=33 \mathrm{~mm}$ ). Data are expressed as deviations from straight-contour pressure values: The right panel represents data from the first quarter cycle of tracking and the left represents data collapsed across two additional cycles of tracking.

contours used in these experiments were larger in magnitude than the optically induced curve used in earlier studies. Given a $37-\mathrm{cm}$ chord length, the curvature produced by a 30-diopter prism at a distance of $.43 \mathrm{~m}$ is quite small. During pilot investigation, when a real curve was constructed with comparable magnitude, subjects had trouble judging its shape reliably with their eyes closed. Hence, larger curves were used initially to establish the finger-tracking pressure effect, especially for haptic inspection.

Clearly, though, the comparison between induced and real curves would have more validity if the curves were equated for magnitude. A final experiment was designed to make this direct comparison. One group of subjects finger-tracked an optically induced curve, while a second group finger-tracked, with eyes open, a real-curved contour which was determined to be equal in magnitude to the induced curve.

\section{EXPERIMENT 4}

\section{Method}

Apparatus and Materials. The pressure transducer and data recording apparatus were the same as those used in the previous experiments. For the induced-curvature condition, the pressure transducer was fitted with the $37-\mathrm{cm}$ straight contour. The contour could be viewed in the enclosure through a variable Risley prism set for no refraction, or set at 30 diopters. The latter condition produced a convex-down induced curve.

For the real-curve group, a steel-rod contour was bent to a curvature with a $37-\mathrm{cm}$ chord length and $11-\mathrm{mm}$ sagitta. The resulting radius of curvature was $1.56 \mathrm{~m}$, which subtended about $124^{\circ}$ of visual angle. This magnitude of curvature was exactly the same as the induced curve since it appeared straight when viewed, suitably oriented, through the 30 -diopter prism (nulling method). Like the induced curve, the real curve was oriented convex down.

Procedure. Two separate groups of subjects were run. One inspected real-curved contours, while the other inspected inducedcurved contours. Both groups practiced finger-tracking straight contours while viewing the contours through the glass aperture (no refraction). The experimental trials were then conducted. All subjects first tracked a straight contour and then a curved contour. Filming began after four preliminary cycles of tracking were completed, and continued for two additional cycles. At the completion of a trial, the subjects were asked to judge whether the contour was curved or straight.

Subjects. Sixteen undergraduate students at Boston College served as volunteer subjects. Eight males and females served in each group.

Data transformations. For the data from the real-curve condition, total finger pressure was computed based on the transduced vertical component of force, radius of curvature, and the specific interval along the contour occupied by the finger. Pressure data from both the real and induced curves were expressed as deviations by subtracting straight contour values from curved contour values.

\section{Results and Discussion}

Pressure deviation data collapsed across four half cycles of tracking are presented for both real and induced curves in Figure 6. Intervals along the abscissa correspond to spatial positions along the contour from the left (Interval 1) to the right (Interval 10) of the subject's median plane. (Preliminary analyses indicated for both groups no effects due to half cycles, and significant effects due to whether tracking was being initiated or terminated, or occurred in a direction roughly away from vs. toward the body.) As can be seen, both pressure profiles are curved and 


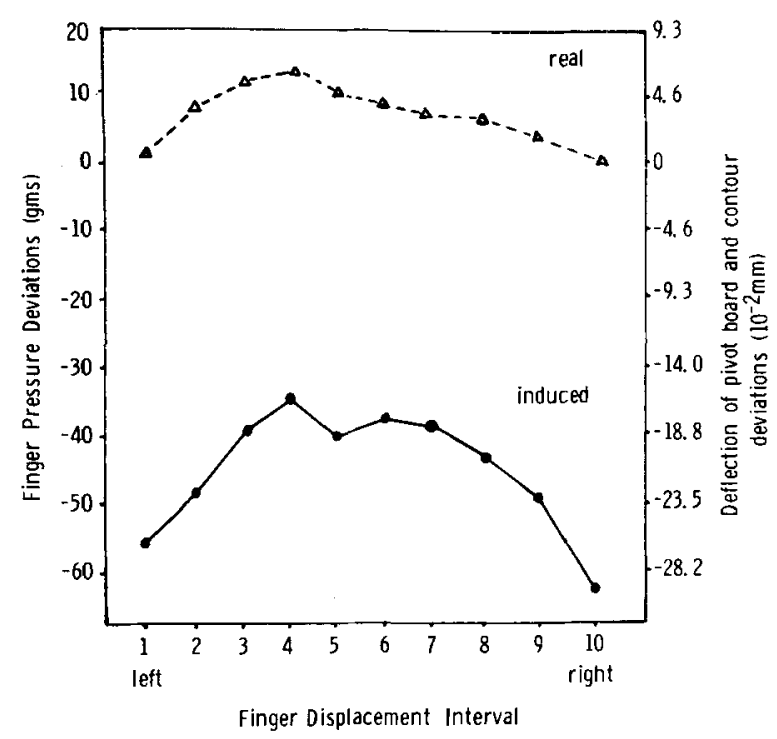

Figure 6. Finger-pressure profiles and corresponding pivotboard displacement resulting from tracking the inside of convexdown real and induced contours (chord $=37 \mathrm{~cm}$, sagitta = $11 \mathrm{~mm}$ ). Data are expressed as deviations from straight-contour pressure values and are collapsed across two cycles of tracking.

opposite in orientation to the contours. A significant quadratic trend component was found to be present in the real curve $[\mathrm{F}(1,126)=4.3, \mathrm{p}<.05]$ as well as in the induced curve $[\mathrm{F}(1,126)=40.6, \mathrm{p}<.001]$ pressure profiles. It was also determined that, while there appears to be a difference, the quadratic component of the induced-curve pressure profile was not significantly larger than the quadratic component of the real-curve pressure profile.

All subjects experienced the straight contour as straight and the curved contours (real or induced) as curved.

The results indicate that pressure profiles from both optically induced and real-curved contours are opposite in orientation to the inspected contours.

\section{GENERAL DISCUSSION}

The results of the four experiments provide consistent evidence that finger-pressure profiles produced during tracking of real curves are opposite in orientation to the contours and the experience of curvature. This proved to be the case whether the eyes were open or closed. Further, the profiles were the result of tracking curvature which was slightly larger in magnitude than contour curvature. The fact that pressure profiles produced during haptic inspection of curvature were the same as those produced during tracking of optically induced curves strengthens the contention that haptic processing is involved in visual capture of curvature. During bimodal perception of real or induced curves, the input to the visual system evidently dominates the haptic response. In visual capture of curvature, then, vision dominates what one does rather than what one feels. However, the precise nature of this visual motor-control process remains to be determined.

One possibility, alluded to in the introduction, is that all visual-haptic bimodal perception of curvature can be viewed as involving sensory conflict. A consequence would be that whether real or induced curvature is inspected, tracking-limb motion would be triggered by a visual image which is larger in curvature than the contour. Resultant finger-pressure profiles would be curved and inversely related to contour curvature. Further, since optically induced curves would represent an enhanced sensory conflict, they would presumably yield larger finger-tracking pressure changes. The results of the fourth experiment seem consistent with this perspective, since curved pressure profiles from induced curves appeared to be larger than those from real curves. However, the between-subjects difference failed to reach statistical significance.

Actually, a more fundamental difficulty is encountered by the sensory conflict account when it is applied to an additional finding of the study. Recall that pressure profiles from real curves were also opposite in orientation to the contour even when vision was not permitted. Thus, sensory conflict does not appear to be a necessary condition for the obtained pressure profiles. Unless it can be demonstrated that the haptic pressure profiles (eyes-closed condition) are the result of a conflict between haptic stimulus information and some internal visual metric of curvature, an alternative to the sensory conflict explanation should be sought to account for the pressure profiles obtained from induced and real curves.

In fact, an alternative explanation becomes apparent by inspecting the finger-tracking data from the eyes-closed condition. The results reveal that two stages of inspection occurred. Initially, subjects tracked through curves slightly smaller in magnitude than the curved contour with resultant increase or decrease in finger pressure depending upon whether the inside or the outside of a curved contour was being inspected. This stage was short-lived, however, and gave way to a stage which involved tracking curves slightly larger in magnitude than the stimulus curve. The size of the smaller or larger tracking curves relative to the contour curve were very small. As can be seen in Figures 3, 4, and 5, the sagittas of tracking curves from both the first quarter cycle and remaining two cycles of tracking were at most only about $0.1 \mathrm{~mm}$ smaller or larger than the sagitta of the contour curve. It is most unlikely that these limbcurvature effects contributed to felt curvature in any absolute way. They did, however, serve to create appreciable pressure changes at the finger. The data from both stages of inspection indicate that from 10 
to $30 \mathrm{~g}$ of pressure change information were available to observers during finger-tracking. This change occurred against an average background of about $100 \mathrm{~g}$ of absolute finger pressure (see Footnote 1). It is difficult to extend findings in the psychophysics literature to the present situation because subjects' fingers were moving and producing pressure changes, but since the Weber ratio for lifted weights is $1 / 40$, and $1 / 30$ for weights placed on the skin, it seems reasonable to assume that pressure change of the magnitude observed during tracking is detectable by humans.

This finding suggests an alternative to sensory conflict as an account of the pressure profiles obtained during finger tracking of induced and real contours. A reasonable case could be made that proximal pressure change is important for haptic perception in the same sense that proximal change is important for visual or auditory perception. During inspection of optically induced or real-curved contours, subjects may have performed incipient limb movement in order to produce pressure change which signified curvature and corresponded to visual information. Pressure alone could not have specified curvature, however, since during haptic inspection pressure change profiles from the two stages of inspection were inversely related. Yet the profiles were presumably capable of signaling the same distal contour slope, since a switch to a different form of tracking occurred. Therefore, if pressurechange information is important and used during haptic perception, its significance must be determined by limb-movement information. ${ }^{3}$ This formulation of course, does not explain why finger-pressure and contour-curvature orientation are inversely related once tracking is underway, but the important point for now is that a haptic system mechanism could account for the pressure profiles from the present experiments, leaving no need to invoke sensory conflict as a necessary condition.

The contention that haptics mediate visual capture of curvature may seem a bit forced, since subjects inspecting induced curvature evidently ignore joint information which could signal straightness. However, pressure produced at the finger not only has an efferent origin, but also is not restricted to a cutaneous effect. Finger pressure could feed back and interact subcutaneously with joint articulations. A consequence would be that the joints could signal curvature even though the tracking limb had not moved through a curved path as large as it would had a real-curved contour been tracked.

In summary, it can be stated that pressure profiles produced during tracking of real-curved contours are curved and opposite in orientation to the contour, whether the eyes are open or closed, and are therefore similar to those obtained during finger-tracking of optically induced curves. Whether the pressure profiles from real curves or optically induced curves are the consequence of sensory conflict, broadly conceived to include all bimodal perception of curvature, or are produced by the functioning of the haptic system, remains to be determined. In either case, the fact that haptic pressure profiles are the same as those produced during finger-tracking of optically induced curves strengthens the contention that haptics mediate visual capture of curvature. Thus, while vision may exert its dominant effect in a sensory conflict situation, by having privileged aecess to central attention, it does not necessarily do so to the exclusion of haptic processing.

\section{REFERENCES}

Coren, S., \& Festinger, L. An alternative view of the "Gibson normalization effect." Perception \& Psychophysics, $1967,2,621-626$.

Easton, R. D. Prismatically induced curvature and fingertracking pressure changes in a visual capture phenomenon. Perception \& Psychophysics, 1976, 19, 201-205.

Gibson, J. J. Adaptation, after-effect and contrast in the perception of curved lines. Joumal of Experimental Psychology, 1933, 16, 1-31.

Gibson, J. J. Observations on active touch. Psychological Review, $1962,69,477.491$.

GiBson, J. J. The senses considered as perceptual systems. Boston: Houghton-Mifflin, 1966.

KOHLER, I. Experiments with goggles. Scientific American, 1962 , 206, 62-72.

Ptaget, J. The mechanisms of perception. New York: Basic Books, 1969.

Posner, M. I., Nissen, M. J., \& Klein, R. M. Visual dominance: An information-processing account of its origins and significance. Psychological Review, 1976, 83, 157-171.

Rock, I. An introduction to perception. New York: Macmillan, 1975.

WINER, B. J. Statistical principles in experimental design (2nd ed.). New York: McGraw-Hill, 1971.

\section{NOTES}

1. The suspension stiffness constant was computed with the use of a sighting microscope to determine pivot-board displacement when weights were applied during calibration. While the pivot board actually displaced through a curved path due to its rearhinging, the magnitude of its displacement was so small across the range of absolute finger pressure applied by subjects $(20-200 \mathrm{~g}$, or $0.1-1.0 \mathrm{~mm}$ of deflection) that its path was assumed to be vertically straight. Thus, displacement measures were expressed in millimeters rather than degrees.

2. If the inside of an arc is finger-tracked this data transformation has the effect of slightly decreasing the pressure differences transduced along the contour. If the outside of an arc is fingertracked, the transformation has the effect of slightly increasing pressure changes. However, the magnitudes of the curvatures used in the present series of experiments were small enough for the effects of the transformations not to be statistically significant. The transformations were nevertheless performed for all experiments so that the data would accurately reflect finger-pressure and limb-movement curvatures.

3. Gibson (1962, 1966) has arrived at a similar conclusion regarding the relation between cutaneous and limb-movement information which exists during haptic inspection.

(Received for publication January 31, 1978; revision accepted May 29, 1978.) 